\title{
LEBESGUE EQUIVALENCE TO FUNCTIONS OF THE FIRST BAIRE CLASS ${ }^{1}$
}

\author{
WILLIAM J. GORMAN III
}

We shall deal with real-valued functions defined on the closed interval $I=[0,1]$.

It has been known for some time that every (Lebesgue) measurable function agrees almost everywhere with some function of the first or second Baire class, and there are examples showing that this statement is no longer true if "second" is deleted. Nevertheless, one might suspect that the class of measurable functions does not, in some sense, differ radically from the first Baire class. Thus one might propose that each measurable function could be transformed by some homeomorphic change of variable into a function that agrees almost everywhere with some function of the first Baire class. Such, however, is not the case. We shall produce a measurable function-one, in fact, with a countable range-for which every homeomorphic change of variable yields either a nonmeasurable function or a measurable function that differs from every function of the first Baire class on a set of positive measure.

This leads to the question: what functions can be transformed by a homeomorphic change of variable into a function that agrees almost everywhere with some function of the first Baire class? We shall show that there are at least two classes of such functions: those possessing the Baire property [1, pp. 354 and 302] and the measurable functions whose range is a finite set.

We shall direct our attention first to the above-mentioned counterexample.

EXAMPLE 1. There is a measurable function $f$ such that, given any homeomorphism $\phi$ of $I$ onto itself, there is no function of the first Baire class that agrees almost everywhere with $f \circ \phi$ ( $\phi$ taken first).

Construction. Let $P_{1}$ be the nowhere-dense perfect set whose complement consists of one interval of length $1 / 4$ located exactly in the middle of $I, 2$ intervals of length $(1 / 4)^{2}$ each located exactly in the middle of one of the 2 remaining intervals, $2^{2}$ intervals of length

Presented to the Society, January 26, 1965 under the title Some notes on the Lebesgue equivalence of real functions; received by the editors August 2, 1965.

1 This work constitutes a part of the author's doctoral dissertation. The research was sponsored by the National Science Foundation through Grant GP-3515. The author gratefully acknowledges the guidance of Professor Casper Goffman. 
$(1 / 4)^{3}$ each located exactly in the middle of one of the $2^{2}$ remaining intervals, and so forth (in analogy with the usual construction of the complement of the Cantor ternary set). The measure of $P_{1}$ is $1 / 2$.

Let $P_{2}$ be the subset of $I-P_{1}$ that meets each component of $I-P_{1}$ in an analog of $P_{1}-\{0,1\}$ (so that the measure of the part of $P_{2}$ in each component is one-half the length of the component).

Continuing in this manner, we produce, for each integer $n>1$, the set $P_{n}$ contained in $I-\left(P_{1} \cup \ldots \cup P_{n-1}\right)$ and meeting each component of the latter in an analog of $P_{1}-\{0,1\}$. Let $P$ denote the union of all the $P_{n}$.

Let $S$ be a set such that neither it nor its complement contains a perfect set [2], and define

$$
Q_{1}=(I-P) \cap S \text { and } Q_{2}=(I-P) \cap(I-S) .
$$

Let

$$
\begin{aligned}
f(x) & =n & & \text { if } x \in P_{n}, \\
& =-1 & & \text { if } x \in Q_{1}, \\
& =-2 & & \text { if } x \in Q_{2} .
\end{aligned}
$$

Verification. Clearly, $f$ is measurable.

Suppose that there is a homeomorphism $\phi$ of $I$ on to itself such that $f \circ \phi$ agrees almost everywhere with some function $g$ of the first Baire class. Then, certainly, $f \circ \phi$ must be measurable. Hence, the sets $\phi^{-1}\left(Q_{1}\right)$ and $\phi^{-1}\left(Q_{2}\right)$, which contain no perfect sets, must have measure 0 ; and so $\phi^{-1}(P)$ must have measure 1 . It follows from the construction of $P$ that, for any given subinterval of $I$, there are an infinite number of $n$ for which $(f \circ \phi)^{-1}(n)$, and therefore $g^{-1}(n)$, meets the subinterval in a set of positive measure. Thus, $g$ must be a Baire 1 function without points of continuity-an impossibility.

In the following theorems, we present two classes of functions that are transformable in the manner indicated above.

THEOREM 1. If a function $f$ possesses the Baire property, there is a homeomorphism $\phi$ of $I$ onto itself such that $f \circ \phi$ agrees almost everywhere with a function of the first Baire class.

Proof. Let $g$ be a semicontinuous function that agrees with $f$ everywhere except on a set $T$ of the first category. If $\phi$ is any homeomorphism of $I$ on to itself such that the measure of $\phi^{-1}(T)$ is 0 , then $f \circ \phi$ agrees almost everywhere with the semicontinuous (and perforce Baire 1) function $g \circ \phi$. The existence of such a homeomorphism has been shown by T. Swiatkowski [3]. 
Since there are nonmeasurable functions that possess the Baire property, Theorem 1 shows that measurability is not a necessary condition for transformability into a function that agrees almost everywhere with a function of the first Baire class.

THEOREM 2. If $f$ is a measurable function whose range is a finite set, then there is a homeomorphism $\phi$ of I onto itself such that $f \circ \phi$ agrees almost everywhere with some function of the first Baire class.

Proof. Denote the range of $f$ by $a_{1}, \cdots, a_{N}$. For $n=1, \cdots, N$, let $S_{n}$ be the set of points of which every neighborhood meets $f^{-1}\left(a_{n}\right)$ in a set of positive measure. Using the superscript ${ }^{\circ}$ to indicate the interior of a set, we denote the (open) components of

$$
\begin{array}{ll}
S_{1}^{\circ} & \text { by } I_{11}, I_{12}, \cdots ; \\
I-S_{1} & \text { by } J_{11}, J_{12}, \cdots ;
\end{array}
$$

and, in general, the (open) components of

$$
\begin{aligned}
& \bigcup_{j=1}^{\infty}\left(S_{i}^{\circ} \cap J_{i-1}\right) \quad \text { by } I_{i 1}, I_{i 2}, \cdots, i=2, \cdots, N ; \\
& \bigcup_{j=1}^{\infty}\left(\left(I-S_{i}\right) \cap J_{i-1}\right) \quad \text { by } J_{i 1}, J_{i 2}, \cdots, i=2, \cdots, N-1 .
\end{aligned}
$$

It is not difficult to see that each $S_{n}$ is closed and that the union of all of them is $I$. Thus for $i=N$, the last expression above is empty, and the union of all the $I_{i j}$, which we shall denote by $G$, is a dense open set. Then, there is a homeomorphism $\phi$ such that the measure of $\phi^{-1}(G)$ is equal to 1 . Since $f^{-1}\left(a_{i}\right) \cap I_{i j}$ always contains an $F_{\sigma}$ set that is uncountable in every subinterval of $I_{i j}$, it is possible to construct $\phi$ so that the measure of $\phi^{-1}\left(f^{-1}\left(a_{i}\right) \cap I_{i j}\right)$ is the same as the measure of $\phi^{-1}\left(I_{i j}\right), j=1,2, \cdots$ and $i=1, \cdots, N$.

Define

$$
\begin{aligned}
& g(x)=a_{i} \quad \text { if } x \in \phi^{-1}\left(I_{i j}\right) \quad \text { for some } j, \\
& =0 \quad \text { otherwise. }
\end{aligned}
$$

The function $g$ is a member of the first Baire class; and $(f \circ \phi)(x)$ $=g(x)$ almost everywhere on each $\phi^{-1}\left(I_{i j}\right)$ and, therefore, almost everywhere on $\phi^{-1}(G)$, which has measure 1 .

Restrictive as this theorem is, it cannot be extended to functions with a countable range. Example 1 serves as a counterexample.

The following example shows that Theorem 2 is not a special case of Theorem 1. 
ExAmple 2. There is a measurable function $h$, not possessing the Baire property, but having a finite range.

Construction. Using the notation of Example 1, we define $h$ to be the characteristic function of $S-P$.

Verification. $h$ is measurable because $I-P$ has measure zero. Since every uncountable Borel set contains a perfect set $[1$, p. 356], both $S$ and $I-S$ meet every subinterval of $I$ in a set of the second category. Thus, since $P$ is of the first category, $h$ is, relative to any residual set, everywhere discontinuous; hence, $h$ cannot possess the Baire property.

There are quite possibly other classes of functions that are transformable in the manner we have discussed; and as an area of investigation we propose the absolutely measurable functions: those functions $f$ such that $f \circ \phi$ is measurable for every homeomorphism $\phi$ of $I$ on to itself.

\section{REFERENCES}

1. H. Hahn, Reele Funktionen, Erster Teil, Chelsea, New York, 1948.

2. W. Sierpinski, Sur un ensemble punctiforme connexe, Fund. Math. 1 (1920), 8.

3. T. Swiatkowski, Sur une transformation d'une fonction measurable en une fonction sommable, Fund. Math. 52 (1963), 1-12.

Purdue University 